\title{
On compatible linear connections with totally anti-symmetric torsion tensor of three-dimensional generalized Berwald manifolds
}

\section{Cs. Vincze ${ }^{1}$ (D)}

Received: 28 March 2019 / Accepted: 19 June 2019 / Published online: 28 June 2019

(c) The Author(s) 2019

\begin{abstract}
Generalized Berwald manifolds are Finsler manifolds admitting linear connections such that the parallel transports preserve the Finslerian length of tangent vectors. By the fundamental result of the theory (Vincze in J AMAPN 21:199-204, 2005) such a linear connection must be metrical with respect to the averaged Riemannian metric given by integration of the Riemann-Finsler metric on the indicatrix hypersurfaces. Therefore the linear connection is uniquely determined by its torsion tensor. If the torsion is zero then we have a classical Berwald manifolds. Otherwise the torsion is a strange data we need to express in terms of quantities of the Finsler manifold. In the paper we are going to give explicit formulas for the linear connections with totally anti-symmetric torsion tensor of three-dimensional generalized Berwald manifolds (Theorem 2). The results are based on averaging of (intrinsic) Finslerian quantities by integration over the indicatrix surfaces. They imply some consequences for the base manifold as a Riemannian space with respect to the averaged Riemannian metric (Theorems 3 and 4). The possible cases are Riemannian spaces of constant zero curvature, constant positive curvature or Riemannian spaces admitting Killing vector fields of constant Riemannian length.
\end{abstract}

Keywords Finsler spaces · Generalized Berwalds spaces · Intrinsic Geometry

Mathematics Subject Classification 53C60 - 58B20

Cs. Vincze is supported by the EFOP-3.6.1-16-2016-00022 project. The project is co-financed by the European Union and the European Social Fund. The work is also supported by TKA-DAAD 307818.

\footnotetext{
$凶$ Cs. Vincze

csvincze@science.unideb.hu

1 Institute of Mathematics, University of Debrecen, Debrecen P.O.Box 400, 4002, Hungary
} 


\section{Introduction}

The notion of generalized Berwald manifolds goes back to Wagner (1943). They are Finsler manifolds admitting linear connections such that the parallel transports preserve the Finslerian length of tangent vectors (compatibility condition). The basic questions of the theory are the unicity of the compatible linear connection and its expression in terms of the canonical data of the Finsler manifold (intrinsic characterization). In case of a classical Berwald manifold admitting a compatible linear connection with zero torsion, the intrinsic characterization is the vanishing of the mixed curvature tensor of the canonical horizontal distribution. In general the intrinsic characterization of the compatible linear connection is based on the so-called averaged Riemannian metric given by integration of the Riemann-Finsler metric on the indicatrix hypersurfaces. By the fundamental result of the theory (Vincze 2005a) such a linear connection must be metrical with respect to the averaged Riemannian metric. Therefore the linear connection is uniquely determined by its torsion tensor. Following Agricola and Friedrich (2004) consider the decomposition

$$
\begin{aligned}
T(X, Y) & :=T_{1}(X, Y)+T_{2}(X, Y), \text { where } \\
T_{1}(X, Y) & :=T(X, Y)-\frac{1}{n-1}(\tilde{T}(X) Y-\tilde{T}(Y) X),
\end{aligned}
$$

$\tilde{T}$ is the trace tensor of the torsion and

$$
T_{2}(X, Y):=\frac{1}{n-1}(\tilde{T}(X) Y-\tilde{T}(Y) X)
$$

In case of $2 \mathrm{D}$ the torsion tensor is automatically of the form (1); see Vincze et al. (2018). If the dimension is at least three then the trace-less part can be divided into two further components

$$
T_{1}(X, Y)=A_{1}(X, Y)+S_{1}(X, Y) \Rightarrow T(X, Y)=A_{1}(X, Y)+S_{1}(X, Y)+T_{2}(X, Y)
$$

by separating the totally anti-symmetric/axial part $A_{1}$. Therefore we have eight possible classes of generalized Berwald manifolds depending on the surviving terms such as classical Berwald manifolds admitting torsion-free compatible linear connections (Szabó 1981) (we have no surviving terms) or Finsler manifolds admitting semisymmetric compatible linear connections (we have no trace-less part) (Vincze 2005b, 2008, 2013) and (Vincze 2017). In the paper we are going to give explicit formulas for the linear connections with totally anti-symmetric torsion preserving the Finslerian length of tangent vectors in case of three-dimensional Finsler manifolds (Theorem 2). The results are based on averaging of (intrinsic) Finslerian quantities by integration over the indicatrix surfaces. They imply some consequences for the base manifold as a Riemannian space with respect to the averaged Riemannian metric. The possible cases are Riemannian space forms of constant zero curvature, constant positive curvature or Riemannian spaces admitting Killing vector fields of constant Riemannian length (Theorems 3 and 4). The Riemannian consequences are simple to prove but they have 
essential influence on the differential topology of the base manifold (Remarks 2 and 3). However, the results are dominated by Theorem 2 as the explicit expression of the only possible compatible linear connection with totally anti-symmetric torsion for a Finsler metric in 3D.

\section{Notations and terminology}

Let $M$ be a differentiable manifold with local coordinates $u^{1}, \ldots, u^{n}$. The induced coordinate system of the tangent manifold $T M$ consists of the functions $x^{1}, \ldots, x^{n}$ and $y^{1}, \ldots, y^{n}$. For any $v \in T_{p} M, x^{i}(v):=u^{i} \circ \pi(v)=p$ and $y^{i}(v)=v\left(u^{i}\right)$, where $i=1, \ldots, n$ and $\pi: T M \rightarrow M$ is the canonical projection.

\subsection{Finsler metrics}

A Finsler metric is a continuous function $F: T M \rightarrow \mathbb{R}$ satisfying the following conditions:

(F1) $F$ is smooth on the complement of the zero section (regularity),

(F2) $F(t v)=t F(v)$ for all $t>0$ (positive homogenity),

(F3) the Hessian

$$
g_{i j}=\frac{\partial^{2} E}{\partial y^{i} \partial y^{j}}, \text { where } E=\frac{1}{2} F^{2}
$$

is positive definite at all nonzero elements $v \in T_{p} M, p \in M$ (strong convexity).

The so-called Riemann-Finsler metric $g$ is constituted by the components $g_{i j}$. It is defined on the complement of the zero section because the second order partial differentiability of the energy function at the origin does not follow automatically: if $E$ is of class $C^{2}$ on the entire tangent manifold $T M$ then, by the positively homogeneity of degree two, it follows that $E$ is quadratic on the tangent spaces, i.e. the space is Riemannian. The Riemann-Finsler metric makes each tangent space (except at the origin) a Riemannian manifold with standard canonical objects such as the volume form $d \mu=\sqrt{\operatorname{det} g_{i j}} d y^{1} \wedge \cdots \wedge d y^{n}$, the Liouville vector field $C:=y^{1} \partial / \partial y^{1}+\cdots+y^{n} \partial / \partial y^{n}$ and the induced volume form

$$
\mu=\sqrt{\operatorname{det} g_{i j}} \sum_{i=1}^{n}(-1)^{i-1} \frac{y^{i}}{F} d y^{1} \wedge \ldots \wedge d y^{i-1} \wedge d y^{i+1} \ldots \wedge d y^{n}
$$

on the indicatrix hypersurface $\partial K_{p}:=F^{-1}(1) \cap T_{p} M(p \in M)$.

\subsection{Generalized Berwald manifolds}

Definition 1 A linear connection $\nabla$ on the base manifold $M$ is called compatible to the Finslerian metric if the parallel transports with respect to $\nabla$ preserve the Finslerian 
length of tangent vectors. Finsler manifolds admitting compatible linear connections are called generalized Berwald manifolds.

Suppose that the parallel transports with respect to $\nabla$ (a linear connection on the base manifold) preserve the Finslerian length of tangent vectors and let $X$ be a parallel vector field along the curve $c:[0,1] \rightarrow M$. We have that

$$
\left(x^{k} \circ X\right)^{\prime}=c^{k^{\prime}} \text { and }\left(y^{k} \circ X\right)^{\prime}=X^{k^{\prime}}=-c^{i^{\prime}} X^{j} \Gamma_{i j}^{k} \circ c
$$

because of the differential equation for parallel vector fields. If $F$ is the Finslerian metric then

$$
(F \circ X)^{\prime}=\left(x^{k} \circ X\right)^{\prime} \frac{\partial F}{\partial x^{k}} \circ X+\left(y^{k} \circ X\right)^{\prime} \frac{\partial F}{\partial y^{k}} \circ X
$$

and, by formula (2),

$$
(F \circ X)^{\prime}=c^{i^{\prime}}\left(\frac{\partial F}{\partial x^{i}}-y^{j} \Gamma_{i j}^{k} \circ \pi \frac{\partial F}{\partial y^{k}}\right) \circ X .
$$

This means that the parallel transports with respect to $\nabla$ preserve the Finslerian length of tangent vectors (compatibility condition) if and only if

$$
\frac{\partial F}{\partial x^{i}}-y^{j} \Gamma_{i j}^{k} \circ \pi \frac{\partial F}{\partial y^{k}}=0(i=1, \ldots, n)
$$

where the vector fields of type

$$
X_{i}^{h^{\nabla}}:=\frac{\partial}{\partial x^{i}}-y^{j} \Gamma_{i j}^{k} \circ \pi \frac{\partial}{\partial y^{k}}
$$

span the associated horizontal distribution belonging to $\nabla$.

Theorem 1 (Vincze 2005a) If a linear connection on the base manifold is compatible to the Finslerian metric function then it must be metrical with respect to the averaged Riemannian metric

$$
\gamma_{p}(v, w):=\int_{\partial K_{p}} g(v, w) \mu=v^{i} w^{j} \int_{\partial K_{p}} g_{i j} \mu\left(v, w \in T_{p} M, p \in U\right) .
$$

Using the so-called Binet-Legendre metric is another possibility to provide a Riemannian environment for the investigations. It has been succesfully used by Troyanov and Matveev (2012), see also Bartelmeß and Matveev (2018). 


\section{Three-dimensional Finsler manifolds admitting compatible linear connections with totally anti-symmetric torsion tensor}

Suppose that $\nabla$ is a compatible linear connection of a three-dimensional generalized Berwald manifold. By Theorem 1, such a linear connection must be metrical with respect to the averaged Riemannian metric (7) given by integration of the RiemannFinsler metric on the indicatrix hypersurfaces. Therefore $\nabla$ is uniquely determined by its torsion tensor. Taking vector fields with pairwise vanishing Lie brackets on the neighbourhood $U$ of the base manifold, the Christoffel process implies that

$$
\begin{aligned}
& X \gamma(Y, Z)+Y \gamma(X, Z)-Z \gamma(X, Y) \\
& \quad=2 \gamma\left(\nabla_{X} Y, Z\right)+\gamma(X, T(Y, Z))+\gamma(Y, T(X, Z))-\gamma(Z, T(X, Y))
\end{aligned}
$$

and, consequently,

$$
\begin{aligned}
\gamma\left(\nabla_{X}^{*} Y, Z\right)=\gamma & \left(\nabla_{X} Y, Z\right)+\frac{1}{2}(\gamma(X, T(Y, Z))+\gamma(Y, T(X, Z)) \\
& -\gamma(Z, T(X, Y)))
\end{aligned}
$$

where $\nabla^{*}$ denotes the Lévi-Civita connection of the averaged Riemannian metric.

Definition 2 The torsion tensor is totally anti-symmetric if its lowered tensor

$$
T_{b}(X, Y, Z):=\gamma(T(X, Y), Z)
$$

belongs to $\wedge^{3} M$.

Corollary 1 If $\nabla$ is a metrical linear connection with totally anti-symmetric torsion then

$$
\nabla_{X}^{*} Y=\nabla_{X} Y-\frac{1}{2} T(X, Y)
$$

and the geodesics of $\nabla^{*}$ and $\nabla$ coincide.

If $\operatorname{dim} M=3$ then $\operatorname{dim} \wedge^{3} M=1$ and, consequently,

$$
T_{b}\left(\frac{\partial}{\partial u^{i}}, \frac{\partial}{\partial u^{j}}, \frac{\partial}{\partial u^{k}}\right)=f \gamma\left(\frac{\partial}{\partial u^{i}} \times \gamma \frac{\partial}{\partial u^{j}}, \frac{\partial}{\partial u^{k}}\right)=f \operatorname{det} \gamma_{i j}
$$

for some local function $f: U \rightarrow \mathbb{R}$, where the orientation is choosen such that the coordinate vector fields represent a positive basis. This means that

$$
\nabla_{X}^{*} Y=\nabla_{X} Y-\frac{f}{2} X \times_{\gamma} Y
$$


Taking the Riemannian energy $E^{*}(v):=\gamma(v, v) / 2$, the Riemann-Finsler metric is $g_{i j}^{*}=\gamma_{i j} \circ \pi$ and the cross product of vertical vector fields is defined by

$$
g^{*}\left(\frac{\partial}{\partial y^{i}} \times g^{*} \frac{\partial}{\partial y^{j}}, \frac{\partial}{\partial y^{k}}\right)=\operatorname{det} g_{i j}^{*}=\operatorname{det} \gamma_{i j} \circ \pi
$$

with bilinear extension. Formula (10) can be written in terms of the induced horizontal structures as follows. Since the horizontal distributions induced by $\nabla^{*}$ and $\nabla$ are spanned by the vector fields

$$
X_{i}^{h^{*}}=\frac{\partial}{\partial x^{i}}-y^{j} \Gamma_{i j}^{* l} \circ \pi \frac{\partial}{\partial y^{l}} \text { and } X_{i}^{h^{\nabla}}=\frac{\partial}{\partial x^{i}}-y^{j} \Gamma_{i j}^{l} \circ \pi \frac{\partial}{\partial y^{l}},
$$

respectively, we have, by formula (10), that

$$
X_{i}^{h^{*}}=X_{i}^{h^{\nabla}}+f \circ \pi V_{i}, \text { where } V_{i}=\frac{1}{2} \frac{\partial}{\partial y^{i}} \times g^{*} C(i=1,2,3) .
$$

\subsection{Three-dimensional Finsler manifolds admitting compatible linear connections with totally anti-symmetric torsion tensor}

Let $M$ be a three-dimensional Finsler manifold admitting a compatible linear connection with totally anti-symmetric torsion tensor. Using the comparison formula (11), the compatibility condition (5) gives that

$$
X_{i}^{h^{*}} E=f \circ \pi V_{i} E(i=1,2,3) \Rightarrow V E=f \circ \pi \sum_{i=1}^{3}\left(V_{i} E\right)^{2},
$$

where the vector field $V$ is defined by the formula $V:=\sum_{i=1}^{3}\left(V_{i} E\right) X_{i}^{h^{*}}$.

Lemma 1 If $\sum_{i=1}^{3}\left(V_{i} E\right)_{v}^{2}=0$ for any $v \in T_{p} M$ then the Finslerian indicatrix $\partial K_{p}$ is a sphere with respect to the averaged Riemannian metric.

Proof Since the infinitesimal rotation represented by the matrix

$$
\left(\begin{array}{ccc}
0 & -v^{1} & v^{2} \\
v^{1} & 0 & -v^{3} \\
-v^{2} & v^{3} & 0
\end{array}\right)
$$

is of rank 2, $\sum_{i=1}^{3}\left(V_{i} E\right)_{v}^{2}=0$ implies that the vector fields $V_{1}, V_{2}$ and $V_{3}$ span the tangent plane to the Finslerian indicatrix at any $v \in \partial K_{p}$. Therefore its Euclidean normal vector field (with respect to $g^{*}$ ) is proportional to $C$. Taking a curve $c:[0,1] \rightarrow$ ว $K_{p}$ we have

$$
0=g_{c(t)}^{*}\left(C \circ c(t), c^{\prime}(t)\right)=\gamma_{p}\left(c(t), c^{\prime}(t)\right)=\frac{1}{2} \gamma_{p}(c, c)^{\prime}(t)
$$


i.e. the Euclidean norm of $c(t)$ is constant. Since the Finslerian indicatrix surface is arcwise connected this means that it is a sphere with respect to the averaged Riemannian metric.

Theorem 2 For a three-dimensional non-Riemannian Finsler manifold, the compatible linear connection with totally anti-symmetric torsion tensor must be of the form

$$
\nabla_{X} Y=\nabla_{X}^{*} Y+\frac{f}{2} X \times_{\gamma} Y
$$

where $\nabla^{*}$ is the Lévi-Civita connection of the averaged Riemannian metric $\gamma$ and the function $f$ is given by

$$
f(p)=\frac{1}{\sigma(p)} \int_{\partial K_{p}} V E \mu
$$

where

$$
V=\sum_{i=1}^{3}\left(V_{i} E\right) X_{i}^{h^{*}}, \quad \sigma(p)=\sum_{i=1}^{3} \int_{\partial K_{p}}\left(V_{i} E\right)^{2} \mu, \quad V_{i}=\frac{1}{2} \frac{\partial}{\partial y^{i}} \times g^{*} C \quad(i=1,2,3)
$$

Proof Since $V E=f \circ \pi \sum_{i=1}^{3}\left(V_{i} E\right)^{2}$ it follows that

$$
\int_{\partial K_{p}} V E \mu=f(p) \sum_{i=1}^{3} \int_{\partial K_{p}}\left(V_{i} E\right)^{2} \mu
$$

If the integrand on the right hand side is zero then, by Lemma $1, \partial K_{p}$ is a Euclidean sphere in $T_{p} M$ with respect to $\gamma$. In case of a generalized Berwald manifolds we have linear parallel transports between the tangent spaces. Since the translates of a quadratic surface are quadratic, this means that the manifold is Riemannian. Otherwise we can divide equation (15) to express the function $f$.

\section{Curvature properties}

Let a point $p \in M$ be fixed and consider the subgroup $G$ of orthogonal transformations with respect to the averaged inner product leaving the indicatrix $\partial K_{p}$ invariant in $T_{p} M$. Such a group is obviously closed in $O(3)$ and, consequently, it is compact. If we have a generalized Berwald manifold then the group $G$ is essentially independent of the choice of $p$ because the parallel translations with respect to the compatible linear connection $\nabla$ makes them isomorphic provided that the manifold is connected. On the other hand $G$ must be finite or reducible unless the manifold is Riemannian; see (Vincze 2018, Remark 5). According to Theorem 1 it follows that Hol $\nabla \subset G$, i.e. the holonomy group of a compatible linear connection is finite or reducible in case of a a non-Riemannian generalized Berwald manifold. Using vector fields $X, Y$ and 
$Z$ with pairwise vanishing Lie-brackets on a neighbourhood $U \subset M$, the comparison formula (13) says that

$$
R(X, Y) Z=R^{*}(X, Y) Z+\frac{1}{2}((X f) Y-(Y f) X) \times_{\gamma} Z+\frac{f^{2}}{4}\left(X \times_{\gamma} Y\right) \times_{\gamma} Z
$$

because of the Jacobi identity

$$
\left(X \times{ }_{\gamma} Y\right) \times{ }_{\gamma} Z+\left(Z \times{ }_{\gamma} X\right) \times{ }_{\gamma} Y+\left(Y \times{ }_{\gamma} Z\right) \times{ }_{\gamma} X=0
$$

and the product rule

$$
\nabla_{X}^{*}\left(Y \times_{\gamma} Z\right)=\left(\nabla_{X}^{*} Y\right) \times_{\gamma} Z+Y \times_{\gamma}\left(\nabla_{X}^{*} Z\right)
$$

Using the vector triple product extension formula

$$
X \times{ }_{\gamma}\left(Y \times{ }_{\gamma} Z\right)=\gamma(X, Z) Y-\gamma(X, Y) Z
$$

it follows that

$$
\gamma(R(X, Y) Y, X)=\gamma\left(R^{*}(X, Y) Y, X\right)-\frac{f^{2}}{4} \operatorname{det}\left(\begin{array}{c}
\gamma(X, X) \gamma(X, Y) \\
\gamma(X, Y) \gamma(Y, Y)
\end{array}\right) .
$$

Remark 1 The curvature tensor of $\nabla$ obviously satisfies the curvature property

$$
R(X, Y) Z=-R(Y, X) Z \text {. }
$$

Property

$$
\gamma(R(X, Y) Z, W)=-\gamma(R(X, Y) W, Z)
$$

also holds because $\nabla$ is metrical with respect to the averaged Riemannian metric $\gamma$. The curvature tensor of $\nabla$ satisfies the Jacobi identity if and only if the function $f$ is constant because

$$
\begin{aligned}
R(X, Y) Z+R(Z, X) Y+R(Y, Z) X \stackrel{(16)}{=} & (X f) Y \times_{\gamma} Z+(Z f) X \\
& \times_{\gamma} Y+(Y f) Z \times_{\gamma} X .
\end{aligned}
$$

Taking the inner product of both sides with $Z$ (for example) it can be easily seen that the left hand side is zero if and only if $Z f=0$ for any vector field $Z$ on the base manifold. To complete the list of the classical curvature properties note that the blocksymmetry and the Jacobi identity are equivalent to each other in 3D for any curvature tensor satisfying (19). It follows by a pure algebraic way (Vincze 2017, Remark 3.11). 


\subsection{The case of finite holonomy group}

Suppose that $G$ is finite and, consequently, the holonomy group of the compatible linear connection is also finite, i.e. its curvature is zero.

Theorem 3 If $M$ is a connected three-dimensional non-Riemannian Finsler manifold admitting a compatible flat linear connection with totally anti-symmetric torsion tensor then the sectional curvature of the averaged Riemannian metric is obviously constant and

- $M$ is a classical Berwald manifold provided that the curvature is zero, or

- $M$ is a proper generalized Berwald manifold provided that the curvature is positive.

Proof Using that $R=0$, formula (17) implies that we have an Einstein metric. As it is known, Einstein metrics in $3 \mathrm{D}$ must be of constant scalar curvature and constant sectional curvature as well.

Remark 2 If $M$ is complete then, by the Killing-Hopf theorem of Riemannian geometry, it follows that the universal cover of $M$ (as a Riemannian space with respect to the averaged Riemannian metric) is isometric to $\mathbb{R}^{3}$ or the Euclidean unit sphere $S^{3} \subset \mathbb{R}^{4}$. Otherwise the manifold (as a non-Riemannian Finsler space) does not admit a compatible flat linear connection with totally anti-symmetric torsion tensor.

\subsection{The case of non-finite reducible holonomy group}

Theorem 4 If $M$ is a connected three-dimensional non-Riemannian Finsler manifold admitting a compatible non-flat linear connection $\nabla$ with totally anti-symmetric torsion tensor then there exists a one-dimensional distribution $\mathcal{D}$ such that

- Any local section of constant length is a covariant constant vector field with respect to $\nabla$,

- Any local section of constant length is a Killing vector field of constant length with respect to the averaged Riemannian metric.

Proof Suppose that $R_{p}(X, Y) \neq 0$ for some point $p \in M$. Since $M$ is a nonRiemannian generalized Berwald manifold it follows that $G$ and, consequently, the holonomy group of $\nabla$ is reducible; see (Vincze 2018, Remark 5). The one-dimensional invariant subspaces constitute a distribution as the point of the manifold is varying. Since the invariant subspaces are translates of each other with respect to $\nabla$ it follows that any local section $\beta$ of constant length must be covariant constant. Therefore

$$
\nabla_{X}^{*} \beta \stackrel{(13)}{=}-\frac{f}{2} X \times{ }_{\gamma} \beta
$$

and, consequently,

$$
[\beta, X]=\nabla_{\beta}^{*} X-\nabla_{X}^{*} \beta=\nabla_{\beta}^{*} X+\frac{f}{2} X \times_{\gamma} \beta=\nabla_{\beta}^{*} X-\frac{f}{2} \beta \times_{\gamma} X,
$$




$$
\begin{aligned}
& \left(\mathcal{L}_{\beta} \gamma\right)(X, Y)=\beta \gamma(X, Y)-\gamma\left(\nabla_{\beta}^{*} X, Y\right)-\gamma\left(\nabla_{\beta}^{*} Y, X\right) \\
+ & \frac{f}{2} \gamma\left(\beta \times_{\gamma} X, Y\right)+\frac{f}{2} \gamma\left(\beta \times_{\gamma} Y, X\right) \\
= & \beta \gamma(X, Y)-\gamma\left(\nabla_{\beta}^{*} X, Y\right)-\gamma\left(\nabla_{\beta}^{*} Y, X\right)=0
\end{aligned}
$$

as was to be proved.

The following example can be considered as a converse of the global version of Theorem 4.

\subsection{An example}

Suppose that $M$ is a connected Riemannian manifold admitting a Killing vector field $\beta$ of unit length. An easy direct computation shows that

$$
\left(\mathcal{L}_{\beta} \gamma\right)(X, Y)=\gamma\left(\nabla_{X}^{*} \beta, Y\right)+\gamma\left(\nabla_{Y}^{*} \beta, X\right)=0,
$$

i.e. the Hesse form $\gamma\left(\nabla_{X}^{*} \beta, Y\right)$ is anti-symmetric. On the other hand $\gamma\left(\nabla_{X}^{*} \beta, \beta\right)=0$ because $\beta$ is of constant length. Therefore

$$
\gamma\left(\nabla_{X}^{*} \beta, Y\right)=-\frac{f}{2} \gamma\left(\beta \times_{\gamma} X, Y\right)
$$

for some function $f$, i.e.

$$
\nabla_{X}^{*} \beta=-\frac{f}{2} \beta \times_{\gamma} X
$$

Taking the metric connection $\nabla$ with torsion $T(X, Y)=f X \times{ }_{\gamma} Y$ it follows, by the comparison formula (13), that $\beta$ is a covariant constant vecor field with respect to $\nabla$. This means that for any vector fields $X$ and $Y$

$$
R(X, Y) \beta=0 \text {, i.e. } R(X, Y) Z=r(X, Y) \beta \times{ }_{\gamma} Z
$$

for some anti-symmetric scalar-valued form $r \in \wedge^{2} M$ because $\beta$ is the vector invariant of the anti-symmetric mapping $Z \mapsto R(X, Y) Z$. By the Ambrose-Singer theorem, the unit component of the holonomy group (the so-called restricted holonomy group) of $\nabla$ is the one-parameter rotational group generated by $\beta$ at each point of the manifold. Taking a point $p \in M$ let us choose a non-qadratic convex revolution surface around the axis of $\beta_{p}$. For an explicit example consider a trifocal ellipsoid (it is a kind of generalized conics instead of the classical conics of the Riemannian geometry) body defined by the equation

$$
\left\|v+\beta_{p}\right\|+\|v\|+\left\|v-\beta_{p}\right\| \leq \text { const. }
$$

the focal set consists of $\pm \beta_{p}, \mathbf{0}$ and the constant is large enough to contain the focal points in the interior of the body to avoid singularities. Using parallel transports with 
respect to $\nabla$ we can extend (21) to each point of the manifold. Note that $\pm \beta_{p}$ in the focal set provide that (21) is invariant under not only the restricted holonomy group but the entire one including possibly reflections about the two-dimensional invariant subspace. Such a smoothly varying family of convex bodies induces a (non-Riemannian) fundamental function $F$ such that it is invariant under the parallel transport with respect to $\nabla$.

Remark 3 Killing vector fields of constant length naturally appear in different geometric constructions such as $K$-contact and Sasakian manifolds (Blair 1976; Belgun et al. 2003; Boyer and Galicki 2000). There are many restrictions to the existence of Killing vector fields of constant length on a Riemannian manifold; for a comprehensive survey see (Berestovskii and Nikonorov 2008): for example, if a compact Riemannian manifold admits such a vector field then its Euler characteristic must be zero in the sense of a Theorem due to Hopf (Berestovskii and Nikonorov 2008, Section 1).

Acknowledgements Open access funding provided by University of Debrecen (DE).

Open Access This article is distributed under the terms of the Creative Commons Attribution 4.0 International License (http://creativecommons.org/licenses/by/4.0/), which permits unrestricted use, distribution, and reproduction in any medium, provided you give appropriate credit to the original author(s) and the source, provide a link to the Creative Commons license, and indicate if changes were made.

\section{References}

Agricola, I., Friedrich, T.: On the holonomy of connections with skew-symmetric torsion. Math. Ann. 328(4), 711-748 (2004)

Bartelmeß, N., Matveev, V.: Monochromatic metrics are generalized Berwald. J. Differ. Geom. Appl. 58, 264-271 (2018)

Blair, D.: Contact Manifolds in Riemannian Geometry. Springer Lectures Notes in Math, vol. 509. Springer, Berlin (1976)

Belgun, F., Moroianu, A., Semmelmann, U.: Symmetries of contact metric manifolds. Geom. Dedic. 101(1), 203-216 (2003)

Berestovskii, V.N., Nikonorov, YuG: Killing vector fields of constant length on Riemannian manifolds. Sib. Math. J. 49(3), 395-407 (2008)

Boyer, C., Galicki, K.: On Sasakian-Einstein geometry. Int. J. Math. 11(7), 873-909 (2000)

Szabó, Z.I.: Positive definite Berwald spaces. Structure theorems on Berwald spaces. Tensor (N. S.) 35(1), 25-39 (1981)

Troyanov, M., Matveev, V.: The Binet-Legendre metric in Finsler geometry. Geom. Top. 16, 2135-2170 (2012)

Vincze, Cs: A new proof of Szabó' s theorem on the Riemann-metrizability of Berwald manifolds. J. AMAPN 21, 199-204 (2005)

Vincze, Cs: On a scale function for testing the conformality of Finsler manifolds to a Berwald manifold. J. Geom. Phys. 54, 454-475 (2005)

Vincze, Cs: On Berwald and Wagner manifolds. J. AMAPN 24, 169-178 (2008)

Vincze, Cs: Generalized Berwald manifolds with semi-symmetric linear connections. Publ. Math. Debr. 83(4), 741-755 (2013)

Vincze, Cs: On a special type of generalized Berwald manifolds: semi-symmetric linear connections preserving the Finslerian length of tangent vectors, "Finsler geometry: New methods and Perspectives". Eur. J. Math. 3(4), 1098-1171 (2017)

Vincze, Cs: Lazy orbits: an optimization problem on the sphere. J. Geom. Phys. 124, 180-198 (2018)

Vincze, Cs., Khosdhani, T., Mehdizadeh, Z., Oláh, M.: Intrinsic characterizations of compatible linear connections of two-dimensional generalized Berwald manifolds, accepted for publication in Commu- 
nications in Math. ArXiv version: On compatible linear connections of two-dimensional generalized Berwald manifolds (2018). arXiv:1808.02644

Wagner, V.: On generalized Berwald spaces. CR Dokl. Acad. Sci. USSR (N.S.) 39, 3-5 (1943)

Publisher's Note Springer Nature remains neutral with regard to jurisdictional claims in published maps and institutional affiliations. 